\title{
BMJ Open Talking about human papillomavirus and cancer: protocol for a patient- centred study to develop scripted consultations
}

\author{
Maggie Hendry, ${ }^{1}$ Di Pasterfield, ${ }^{1}$ Richard Adams, ${ }^{2}$ Mererid Evans, ${ }^{2}$ Alison Fiander, ${ }^{3}$ \\ Michael Robling, ${ }^{4}$ Christine Campbell, ${ }^{5}$ Matthew Makin, ${ }^{6}$ Simon Gollins, ${ }^{7}$ \\ Julia Hiscock, ${ }^{1}$ Sadia Nafees, ${ }^{1}$ Marie-Jet Bekkers, ${ }^{4}$ Jan Rose, ${ }^{8}$ Olwen Williams, ${ }^{9}$ \\ Margaret Stanley, ${ }^{10}$ Clare Wilkinson ${ }^{1}$
}

To cite: Hendry M, Pasterfield D, Adams R, et al. Talking about human papillomavirus and cancer: protocol for a patient-centred study to develop scripted consultations. BMJ Open 2016;6:e011205 doi:10.1136/bmjopen-2016011205

- Prepublication history and additional material is available. To view please visit the journal (http://dx.doi.org/ 10.1136/bmjopen-2016011205).

Received 20 January 2016 Revised 8 February 2016 Accepted 9 February 2016

CrossMark

For numbered affiliations see end of article.

Correspondence to

Maggie Hendry;

m.hendry@bangor.ac.uk

\section{ABSTRACT}

Introduction: Persistent infection with sexually transmitted, high-risk human papillomavirus (HPV) types is the cause of all cervical cancers and some anogenital and oropharyngeal cancers. HPV is an extremely common asymptomatic infection but little known and poorly understood by the public. Patients with HPV-related cancers have new and challenging information needs due to the complex natural history of HPV and the stigma of sexual transmission. They may ask questions that are outside the remit of the traditional cancer consultation, and there is a lack of guidance on how to counsel them. This study aims to fulfil that need by developing and testing cancer sitespecific scripted consultations.

Methods and analysis: A synthesis of findings generated from previous work, a systematic review of information-based interventions for patients with HPVrelated cancers, and interviews with cancer clinicians will provide the evidence base underpinning provisional messages. These will be explored in three phases of face-to-face interviews with 75-90 purposively selected patients recruited in cancer clinics to: (1) select and prioritise the most salient messages, (2) phrase the messages appropriately in plain English and, (3) test their acceptability and usefulness. Phases 1 and 2 will draw on card-sorting methods used in website design. In phase three, we will create cancer site-specific versions of the script and test them using cognitive interviewing techniques.

Ethics and dissemination: The study has received ethical approval. Findings will be published in a peerreviewed journal. The final product will be cancerspecific scripted consultations, most likely in the form of a two-sided information sheet with the most important messages to be conveyed in a consultation on one side, and frequently asked questions for later reading on the reverse. However, they will also be appropriate and readily adaptable to web-based uses.

\section{BACKGROUND}

Nearly all sexually active men and women are exposed to human papillomavirus (HPV)
Strengths and limitations of this study

- Human papillomavirus (HPV) infection is an area of cancer communication which is new for patients and healthcare professionals: we address an urgent need for timely and accurate information.

- Patients will take an active role in developing scripted consultations about HPV-related cancers that are informative, reassuring and important to them.

- The resulting scripts, developed for use in consultations, will be readily modifiable for website content and generalisable beyond UK NHS settings.

- Participants will not be asked to identify their sexual orientation; therefore, there is likely to be some bias towards heterosexuals in the sample.

- Owing to the comparative rarity of penile cancer, we will not seek to include this cancer in the sample.

at some stage in their lives; in most people, it does not cause health problems. HPVs that infect the anogenital tract fall into two broad groups: those that cause warts (low-risk) and those associated with cancer (high-risk). Persistent infection with high-risk HPV types causes all cervical cancer, ${ }^{1}$ most vulvar, vaginal $^{2}$ and anal cancer, ${ }^{3}$ approximately half of penile cancers, ${ }^{4}$ as well as an increasing subset of oropharyngeal cancers, ${ }^{56}$ and HPV is also implicated in cancer precursor conditions in the cervix, anus, vulva and vagina. In some instances, HPV status will determine the approach to cancer treatment. ${ }^{7}$

The rising number of HPV-related cancers is a major public health issue. The concept of a virus causing cancer is frightening. The association of cervical precancer with HPV has clear psychosocial adverse effects. ${ }^{9}$ 
Health professionals must be prepared to discuss HPV status because affected patients may want to know the cause of their condition and may question the implications for their sexual partners. ${ }^{10} 11$ Although discussions about HPV between patients and health professionals are becoming more common in cervical disease, patients express concern about the stigma attached to sexual transmission. ${ }^{12-14}$ There is little rigorous research into how clinicians communicate with patients for the other cancers associated with HPV.

Patients and the public know very little about HPV. Despite the introduction of HPV vaccination in schools, and HPV testing within cervical screening programmes in the UK and several other countries, systematic reviews demonstrate consistently poor knowledge and lack of awareness that HPV is a sexually transmitted causative factor for cervical cancer. ${ }^{9} 15$ Furthermore, women who are found to be HPV positive during cervical screening experience distress, anxiety and a notable lack of understanding. ${ }^{9}$ Healthcare professionals do not know enough about HPV-associated cancers, a developing area of research where there are still many uncertainties and good quality patient information is lacking; they have difficulty initiating discussions about HPV and sexual matters with patients, and find it challenging to explain HPV infection; they describe it as a 'can of worms' ${ }^{16}$ Addressing stigma and psychosexual information needs, in addition to explaining the nature of HPV infection and the link between HPV and cancer, requires the development of high-quality, clear and understandable educational materials and messages.

A 'counselling paradigm' has been proposed, but a limitation of this approach is the lack of studies where patient input has informed education and counselling strategies. ${ }^{17}$ Scripted consultations have been used effectively to deliver patient-sensitive, evidence-based information about difficult scientific subjects. ${ }^{18}$ Written material is designed to educate both healthcare professionals and patients, and improve health outcomes. Our methodological approach fits with the Cochrane Consumers and Communication Review Group's 'The Knowledgeable Patient', ${ }^{19}$ a summary of health benefits from improving patient and public/ professional interactions by integrating systematic review evidence with patient and public views, as well as the co-production principle embedded in NHS Wales' 'Prudent Healthcare'. ${ }^{20}$ We will also draw on the resources and recommendations for producing high-quality patient information outlined by the Patient Information Forum. ${ }^{21}{ }^{22}$ We will generate novel findings by intentionally including patient perspectives into a proposed strategy to provide timely and accurate information on the role of HPV infection in cancer, an area of cancer communication which is relatively new for patients, the general public and many healthcare professionals.

\section{AIMS AND OBJECTIVES}

\section{Overall aim}

To develop and field test evidence-based cancer sitespecific versions of a scripted HPV consultation, including 'take-home' information for patients diagnosed with HPV-related cancer. These scripts will contain the most salient points not to miss, rather than verbatim wording for a consultation. They will be in a format that is suitable for delivery to patients by cancer clinicians; however, they could be readily adapted to provide web-page content for information providers such as NHS Choices, Cancer Research UK and Macmillan. They will be designed to reduce anxiety and increase HPV knowledge in patients with specific cancers.

\section{Objectives}

- To conduct a systematic review of information-based interventions for patients with HPV-related cancers

- To conduct telephone interviews with clinicians to identify their views about, and current practice in, talking about HPV with their patients

- To develop a list of appropriate messages based on previous work (the HPV Core Messages study), the systematic review and the clinician interviews

- To conduct face-to-face exploratory interviews with patients diagnosed with cervical, vulval, vaginal, anal or oropharyngeal cancers to select and prioritise the most salient messages and refine the content and phrase the messages in language that is easy to understand

- To set the resulting messages in scripts that provide take-home messages, which participants believe are succinct, salient and address their concerns, then test their acceptability and usefulness in cognitive debrief interviews with patients and further refine as necessary.

\section{RESEARCH PLAN-METHODS AND ANALYSIS Developing evidence-based draft messages}

Between 2008 and 2011, we developed informational messages pertinent to HPV vaccination and HPV testing in the context of cervical cancer prevention (the HPV Core Messages study). The evidence base for these messages was derived from systematic reviews of people's views about $\mathrm{HPV}$ vaccination ${ }^{15}$ and testing, ${ }^{9}$ qualitative interviews with vaccination-aged girls and their parents, ${ }^{23}$ and with women in the cervical screening programme, and UK-wide surveys of health professionals and the public (mainly information needs). An expert panel and systematic review of reviews underpinned the scientific content of the messages. A subset of these messages is appropriate to be adapted for patients with a diagnosis of HPV-related cancer and this will be our starting point for developing a list of generic and cancer site-specific messages. We will create a supporting evidence matrix, derived from a new systematic review and clinician interviews, and scrutinised by experts including 
specialists in virology and epithelial pathology, and genitourinary medicine, as well as cancer specialists and a patient representative, to ensure that the messages are relevant and accurate.

\section{Systematic review}

We will search Medline, PsycInfo, Embase, Cinahl, Web of Knowledge and The Cochrane Library for studies of any design with a focus on informing patients about any HPV-related cancer. A search strategy developed for Medline will be adapted for the other databases (see online supplementary file 1). Participants could be patients or clinicians; the intervention or topic of interest is information delivered in any format or medium (eg, written leaflets, web pages, videos, consultations). Two reviewers will carry out screening, study selection and quality appraisal; disagreements will be resolved by discussion. Study quality will be appraised using the Critical Appraisal Skills Programme (CASP) tools appropriate to study type. Data, including study details, setting, population, methods, etc, will be extracted into predefined forms. We will present the review findings in a table, accompanied by a narrative summary description and explanation. Any new messages, indicated by the review findings, and their supporting evidence will be added to the evidence matrix.

\section{Clinician interviews}

We will conduct 35-40 telephone interviews with healthcare professionals in Wales, covering all relevant clinical roles (surgeons, oncologists and cancer nurse specialists) and cancer sites (oropharyngeal, gynaecological, anal, penile). We will utilise a topic guide focusing on current practice in consultations with patients including: whether they initiate conversations about HPV; what factors influence the way they approach the topic; what sort of explanations they give; what questions patients commonly ask and what concerns they express; what works well in conversations about HPV; what areas of knowledge are difficult to explain or have the potential to increase patients' concerns. Interviews will be audio recorded and transcribed. New or unforeseen issues that arise during the interviews may be added to the topic guide and further explored in succeeding interviews. We will read the transcripts to familiarise ourselves with the data, which will then be analysed using framework-based synthesis. ${ }^{24}$ We will synthesise the data into a matrix format, structured by the messages identified in the HPV core messages study, and allow for additional unforeseen emerging themes (and potential new messages) to be incorporated. This approach will allow teamwork, transparency and an audit trail of the evolution of the study findings. We will be influenced by the 'best fit framework synthesis' used by Carroll et $a l^{25}$ in integrating the synthesised interview data with the core messages developed in previous research.
Patient interviews

\section{Participants}

We will conduct 75-90 face-to-face interviews with patients in three phases. By recruiting participants in two study centres (North and South Wales), we will be able to include people from rural, semirural and urban settings, and different socioeconomic groups, as well as patients with different HPV-related cancer diagnoses, male and female (where appropriate), and of varied ages. The purposive sample aims and rationale are to achieve an even spread of recruitment across cancer sites, although we will not attempt to recruit patients with penile cancer as this is a very rare condition. Although cases of cervical cancer are most numerous, these patients are likely to be better informed about HPV through the cervical screening programme; potentially, we have more to learn from patients with other cancer types. Since men and women may have different information/communication needs and preferences, we are aiming for about two-thirds male in the cancers that affect both genders to help redress the balance against women with HPV-related gynaecological cancers. Finally, we will aim for more participants in the first two phases of interviews because there is potential in these phases to add to or modify the messages, whereas in the third phase we will test the final 'product'.

Eligible interview participants will be adults who have full mental capacity, are aware of their cancer diagnosis and have been informed by their cancer clinician of its possible connection with HPV and the fact that HPV is sexually transmitted. Patients may be at any stage from diagnosis through follow-up; however, those who are medically unfit to participate or without mental capacity to give informed consent will be excluded. Eligible patients will be approached by their cancer clinician, who will briefly explain the study and give a recruitment pack containing an invitation letter, information sheet and reply slip. Patients will respond directly to the research team, indicating whether they would like to take part; written consent will be obtained at the time of the interview.

Interviews, conducted by experienced qualitative researchers, will last 60-90 min and will take place in participants' homes, university or hospital locations according to convenience and participants' preferences. While the content and style of each phase of interviews will be quite different, all will begin with some background information including a brief discussion of the participants' current and past relationship status, stage in the cancer journey, when and how they first heard about HPV and the sources of their information. All participants will be asked a health literacy question. ${ }^{26}$ The plans for conducting each phase of interviews are as follows: in each case, the methods described will be piloted on volunteers from the North East group of the North Wales Cancer Network Patient Forum, and further refined if necessary. 


\section{Phase 1: Selecting and prioritising the messages}

\section{Method}

Card-sorting methods used in website design ${ }^{27-29}$ will be used to select and prioritise the most important messages for clinicians to discuss with their patients, and identify which are of lesser importance and possibly suitable as take-home information. Participants will be presented with around 20 cards, each printed with a factual statement that is either applicable to all HPV-related cancers or to the participant's specific cancer. The cards will be presented in no particular order (shuffled) and participants will be given time to read the messages and select up to five that they consider to be most important. They will be encouraged to comment on their choices, talk through the thinking process by which they reach their decisions, and ask questions if they are unsure of any statement's meaning or relevance. In collaboration with the participant, the interviewer will use coloured stickers to mark the participants' choices and may also write their (participants') comments on the cards. Comments may be recorded verbatim or summarised (depending on length), and the accuracy of summarised versions verified by the participant. Blank cards will be supplied for any additional messages participants think should be included, or questions they think are not addressed. As soon as possible after the interview, the researcher will transfer any data recorded on the cards to a data-recording sheet, and record their own reflections on the interview. The interviews will be audio recorded to aid completion of the data sheets.

\section{Analysis}

We will collate the written responses, and analysis will be a combination of vote-counting of the messages identified as most and least important, and a descriptive content analysis of the collated comments. Content analysis is an appropriate method for "capturing and interpreting common-sense, substantive meanings in the data" 30 ; we will organise the data in a matrix and look for themes and relationships that help to explain which participants prioritise which messages, and why. ${ }^{31}$ The study advisory group will meet to review and discuss the analysed data and make a final selection of the most important messages. They will also discuss whether new messages suggested by participants (if any) should be included.

\section{Phase 2: Phrasing and framing the messages \\ Method}

In phase 2, a second group of participants will comment on the language and tone of the messages. The researcher will explain that the meaning may not be altered (since all the messages are evidence-based); however, the wording may be changed if necessary, for example, to clarify the meaning, soften the tone or simplify the language. The messages will be presented, one at a time, on printed cards similar to those used in phase 1, allowing as much time for thought and discussion as individuals want. Audio recordings will be made and suggested changes in wording, participants' comments and interviewers' reflections recorded on a data sheet as before.

\section{Analysis}

Proposed changes and comments for each message will be collated and reviewed by the study advisory group. The group will meet and discuss the merit of proposed changes and adjudicate between competing suggestions, ensuring that they are framed according to the aim of the message (informative, explanatory, reassuring, etc) and that the underlying meanings remain intact. The final versions will be reached by consensus.

\section{Phase 3: Testing the scripts}

Method

We will create versions of the scripts for each cancer site. The format will most likely be a two-sided information sheet. On the first side will be the information deemed most important in the process described above, while the second side will be framed as 'further information' or 'frequently asked questions'. We will refer to the Patient Information Forum's online 'Toolkit' for guidance with regard to layout and use of images, graphics, etc, ${ }^{21}$ and we will present the scripts at a meeting of the North Wales Cancer Patient Forum for additional feedback on the layout and content. In this phase, we will test the acceptability and usefulness of the scripts through cognitive debrief interviews and further refine them as necessary.

Page 1 of the scripts will be read with individual patients, by a researcher, as though in a consultation. The researcher will provide explanations and clarifications as requested by the interviewee. Then the patient will read page 2 to him/herself, as though at home. This will be followed by a cognitive interview to assess their understanding of the scripts. Cognitive interviewing allows assessment of (1) how patients understand messages, (2) how such messages are used to draw on relevant attitudes and existing knowledge and (3) how this informs their thinking. ${ }^{32}$ We will use this iterative process to identify and modify any problem areas in the scripts. Cognitive interviewing generally involves probing and think-aloud techniques to assess survey item adequacy using structured interview schedules. ${ }^{32}{ }^{33}$ We will adapt this methodology for developing information messages. Cognitive interviews will be audio recorded for analysis.

\section{Analysis}

Analysis of the cognitive interviews will be descriptive and focus on the attributes of the scripts and information sheets that compromise understanding. Depending on the actual quantity of information (messages) within the script, interview schedules may prioritise components thought to be less accessible to patients. Analysis will aim to summarise observations for such messages, but will also allow for other emergent issues elsewhere. 
Coding of interview observations will emerge from the data set. Of particular interest will be alternate formulations of messages suggested in the interview by either the patient or the interviewer, which may inform message revision. Results will be presented as message level descriptive summaries of observed problems suitably categorised and providing diagnostic information to guide revision.

\section{Outcomes}

The final product will be a family of novel tailored, scripted consultations that are framed and contextualised and field tested on patients as offering messages that are succinct, salient, reassuring and relevant. Each scripted consultation will comprise key information phrased in plain language for the clinician to use in their consultation, accompanied by further information for the patient to read, the whole to be printed on one sheet of paper for the patient to take home. The messages will be readily adaptable to other formats and purposes such as web-based educational materials for clinicians and informational materials for patients.

\section{DISCUSSION}

The main challenge for this study is that there is no 'standard' methodology for this type of work. We have taken a pragmatic approach and adapted methods from other contexts (card-sorting from website design, cognitive interviewing from survey design), which may require further modification as the study progresses. However, we consider the innovative study design to be one of its strengths. Another is that all the messages will be based on at least two sources of evidence, supporting the accuracy of the statements and the need for the information. As well as having a patient representative on our advisory group and involving members of the North Wales Cancer Network Patient Forum, the participants in the study will be actively involved in co-producing the scripts, further ensuring their usefulness and appropriateness.

In a study to develop materials to promote informed choice in the context of breast cancer screening, tensions were reported between the professional experts and the women taking part in the study. ${ }^{34}$ There was disagreement over the appropriate level of detail, particularly where the science is uncertain, and the communication of quantitative information. In our study, we see the sensitivity of the information as the key problem, and here the patient perspective is paramount; we have much less focus on the communication of quantitative information and risk. However, if tensions do arise between the patient and professional perspectives, we will consider testing alternative versions of the scripts in the phase three cognitive debrief interviews.

The interviews will focus on the information we are developing rather than experiences of individual participants, though experiences will no doubt inform individuals' responses. In keeping with a non-judgemental attitude and the approach that all sexually active adults are susceptible to HPV infection, we will avoid asking questions about individuals' sexual orientation or behaviour. Therefore, we will be unable to stratify by sexual orientation, and there is likely to be some bias towards heterosexuals in the samples. However, if any participants identify themselves as lesbian, gay, bisexual or transgender, we will explore that perspective and endeavour to ensure a range of participants in terms of age, gender, socioeconomic status, cancer site and health literacy. Owing to their rarity, we decided not to recruit patients with penile cancer, but we anticipate that their needs for information about HPV will not be dissimilar from patients with other HPV-related cancers and we will look for differences between groups that might shed light on this issue.

The resulting scripts should be generalisable beyond the UK NHS setting, as these difficult conversations about HPV-related cancers are based on global literature and will be easy to modify for other settings. Our intention is to evaluate this approach in terms of effectiveness and cost-effectiveness of this approach in a future pragmatic randomised trial.

\author{
Author affiliations \\ ${ }^{1}$ North Wales Centre for Primary Care Research, Bangor University, Wrexham, \\ UK \\ ${ }^{2}$ Velindre Cancer Centre, Cardiff, UK \\ ${ }^{3}$ Royal College of Obstetricians and Gynaecologists, London, UK \\ ${ }^{4}$ South East Wales Trials Unit, Centre for Trials Research, Cardiff University, \\ Cardiff, UK \\ ${ }^{5}$ Usher Institute for Population Health Sciences and Informatics, The \\ University of Edinburgh, Edinburgh, UK \\ ${ }^{6}$ The Pennine Acute Hospitals NHS Trust, Manchester UK \\ ${ }^{7}$ North Wales Cancer Treatment Centre, Betsi Cadwaladr University Health \\ Board, Rhyl, UK \\ ${ }^{8}$ Patient representative, Gloucester, UK \\ ${ }^{9}$ Department of Sexual Health, Betsi Cadwaladr University Health Board, \\ Wrexham, UK \\ ${ }^{10}$ Department of Pathology, University of Cambridge, Cambridge, UK
}

Acknowledgements The authors acknowledge the help of Professor Peter Saseini who contributed to the design of this study as a co-applicant to the funding, and Dr Amanda Tristram who contributed to the design of patient interviews.

Contributors $\mathrm{CW}$ is the Chief Investigator and conceived the original idea for the research. MH, DP, RA, ME, AF, MR, CC and MM are coapplicants and together with $\mathrm{CW}$ designed the study. JH and SN developed participant recruitment materials and sought ethical and $R \& D$ approval. All authors regularly attend meetings of the Study Advisory Group and have contributed to further development of the protocol. JR represents the patient perspective; OW advises on sexual health, MS advises on virology/pathology of HPV. RA, $\mathrm{ME}, \mathrm{AF}$ and $\mathrm{SG}$ are involved in patient recruitment. $\mathrm{MH}$ and $\mathrm{M}-\mathrm{JB}$ are conducting patient interviews. MH and DP drafted the paper; all authors read, commented and were involved in subsequent redrafts and all have read and approved the final version.

Funding This study is funded by the Welsh Government through Health and Care Research Wales' Research for Patient and Public Benefit programme (Grant No. RfPPB 2012-1005).

Competing interests None declared.

Ethics approval The study started in July 2014 and is due to complete at the end of June 2016. Data collection is ongoing at the time of the writing. It has received approval from the Wales Research Ethics Committee (14/WA/0039) and NHS Research and Development (IRAS: 143724). 
Provenance and peer review Not commissioned; externally peer reviewed.

Open Access This is an Open Access article distributed in accordance with the Creative Commons Attribution Non Commercial (CC BY-NC 4.0) license, which permits others to distribute, remix, adapt, build upon this work noncommercially, and license their derivative works on different terms, provided the original work is properly cited and the use is non-commercial. See: http:// creativecommons.org/licenses/by-nc/4.0/

\section{REFERENCES}

1. Lowy DR, Solomon D, Hildesheim A, et al. Human papillomavirus infection and the primary and secondary prevention of cervical cancer. Cancer 2008;113(7 Suppl):1980-93.

2. Insinga RP, Liaw KL, Johnson LG, et al. A Systematic review of the prevalence and attribution of human papillomavirus types among cervical, vaginal, and vulvar precancers and cancers in the United States. Cancer Epidemiol Biomarkers Prev 2008;17:

1611-22.

3. Oon SF, Winter DC. Perianal condylomas, anal squamous introepithelial neoplasms and screening: a review of the literature. J Med Screen 2010;17:44-9.

4. Backes DM, Kurman RJ, Pimenta JM, et al. Systematic review of human papillomavirus prevalence in invasive penile cancer. Cancer Causes Control 2009;20:449-57.

5. Mannarini L, Kratochvil V, Calabrese L, et al. Human Papilloma Virus (HPV) in head and neck region: review of literature. Acta Otorhinolaryngol Ital 2009;29:119-26.

6. Joseph AW, Pai SI. Human Papillomavirus and the shifting trends in head and neck cancer. Ramaswamy Govindan, Ed. In: ASCO 2011 educational book. Alexandria, Virginia, USA: American Society for Clinical Oncology, 2011:212-16.

7. Marur S, D'Souza G, Westra WH, et al. HPV-associated head and neck cancer: a virus-related cancer epidemic. Lancet Oncol 2010;11:781-9.

8. Baxi SS, Shuman AG, Corner GW, et al. Sharing a diagnosis of HPV-related head and neck cancer: the emotions, the confusion, and what patients want to know. Head Neck 2013;35: 1534-41.

9. Hendry M, Pasterfield D, Lewis $\mathrm{R}$, et al. Are women ready for the new cervical screening protocol in England? A systematic review and qualitative synthesis of views about human papillomavirus testing. Br J Cancer 2012;107:243-54.

10. Evans M, Powell NG. Sexual health in oral oncology: breaking the news to patients with human papillomavirus-positive oropharyngeal cancer. Head Neck 2014;36:1529-33.

11. Finnigan JP, Sikora AG. Counseling the patient with potentially HPV-related newly diagnosed head and neck cancer. Curr Oncol Rep 2014;16:375.

12. Daley EM, Vamos CA, Wheldon CW, et al. Negative emotions and stigma associated with a human papillomavirus test result: a comparison between human papillomavirus-positive men and women. J Health Psychol 2015;20:1073-82.

13. Dyer K. From Cancer to sexually transmitted infection: explorations of social stigma among cervical cancer survivors. Hum Organ 2010;69:321-30.

14. Shepherd MA, Gerend MA. The blame game: cervical cancer, knowledge of its link to human papillomavirus and stigma. Psychol Health 2014:29:94-109.

15. Hendry M, Lewis R, Clements A, et al. "HPV? Never heard of it!": a systematic review of girls' and parents' information needs, views and preferences about human papillomavirus vaccination. Vaccine 2013;31:5152-67.

16. McSherry LA, Dombrowski SU, Francis JJ, et al. 'It's a can of worms': understanding prim ary care practitioners' behaviours in relation to HPV using the theoretical domains framework. Implement Sci 2012;7:73.

17. Chu A, Genden E, Posner M, et al. A patient-centered approach to counseling patients with head and neck cancer undergoing human papillomavirus testing: a clinician's guide. Oncologist 2013;18:180-9.

18. Forbes $L J L$, Linsell $L$, Atkins $L$, et al. A promoting early presentation intervention increases breast cancer awareness in order women after 2 years: a randomised controlled trial. BMJ 2011;105:18-21.

19. Hill S, ed. The knowledgeable patient: communication and participation in health. Wiley-Blackwell Online, 2011

20. Public Health Wales. Making prudent healthcare happen. 2014 http://www.prudenthealthcare.org.uk/principles/ (accessed 20 Nov 2015).

21. Patient Information Forum. PIF Toolkit. http://www.pifonline.org.uk/ toolkit/ (accessed 12 Aug 2015).

22. Patient Information Forum. Guide to appraising health information. 2015. http://www.pifonline.org.uk/wp-content/uploads/2014/11/ PIF-Guide-Appraising-Health-Information-2010.pdf (accessed 28 Jan 2015).

23. Henderson L, Clements A, Damery S, et al. 'A false sense of security'? Understanding the role of the HPV vaccine on future cervical screening behaviour: a qualitative study of UK parents and girls of vaccination age. J Med Screen 2011;18:41-5.

24. Dixon-Woods M. Using framework-based synthesis for conducting reviews of qualitative studies. BMC Med 2011:9:39.

25. Carroll C, Booth A, Cooper K. A worked example of "best fit" framework synthesis: a systematic review of views concerning the taking of some potential chemopreventive agents. BMC Med Res Methodol 2011;11:29.

26. Morris NS, MacLean CD, Chew LD, Littenberg B, et al The Single Item Literacy Screener: evaluation of a brief instrument to identify limited reading ability. BMC Fam Pract 2006;7:21

27. William H. Card sorting. In: Soegaard M, Dam RF, eds. The encyclopedia of human-computer interaction. 2nd edn. Aarhus, Denmark: The Interaction Design Foundation, 2014. https://www. interaction-design.org/literature/book/the-encyclopedia-of-humancomputer-interaction-2nd-ed/card-sorting (accessed 12 Apr 2016).

28. Tullis T. How Many Users Are Enough for a Card-Sorting Study? Tom Tullis_Fidelity Investments (tom.tullis @ fmr.com) Larry WoodBrigham Young University (WoodL@ byu.edu). http://home.comcast. net/ tomtullis/publications/UPA2004CardSorting.pdf (accessed 18 Sep 2015).

29. Useability.gov. Improving the user experience: How to and tools. http://www.usability.gov/how-to-and-tools/methods/card-sorting.html (accessed 14 Jun 2015).

30. Spencer L, Ritchie J, O'Connor W. Analysis: practices, principles and processes. In: Ritchie J, Lewis J, eds. Qualitative research practice. A guide for social science students and researchers. London: Sage, 2003:199-218.

31. Silverman D. Doing qualitative research. A practical handbook (second edition). 2nd edn. London: Sage, 2005.

32. Willis GB. Cognitive interviewing: a tool for improving questionnaire design. Thousand: Oaks Sage, 2005.

33. Jobe JB. Cognitive psychology and self-reports: models and methods. Qual Life Res 2003;12:219-27.

34. Forbes LJ, Ramirez AJ, Expert group on Information about Breast Screening, Offering informed choice about breast screening. J Med Screen 2014;21:194-200. 